\title{
Length measurement by means of a grid
}

\author{
RLE MCINTYRE, J PIRIS* \\ From the John Radcliffe Hospital, Oxford
}

SUMMARY Experiments were performed which demonstrate some of the length-measuring characteristics of a grid consisting of two sets of parallel lines crossing each other at right angles. If lines of various shapes are repeatedly superimposed at random on such a grid, the mean number of intersections each of these lines makes with the lines of the grid is directly proportional to the length of the line. There is a property of two-dimensional curves, which may be called directional bias, the presence of which in a test curve will increase the variability of the number of intersections made with the grid lines and skew the frequency distribution curve to the right. This effect is markedly reduced by taking the mean of three measurements if the second and third are taken with the grid rotated $30^{\circ}$ and $60^{\circ}$ respectively from its original position in relation to the test curve. Information gained from these experiments can be applied to stereological problems such as the estimation of particle densities in histological sections.

A convenient method of measuring the length of lines of various shapes is to count the number of intersections they make with lines on a test grid. One such grid, consisting of two sets of parallel lines, half an inch apart at right angles to each other, was to be used to measure the length of tissue profiles in the quantification of cells in tissue sections. Some simple experiments were devised to demonstrate some of the length-measuring characteristics of this grid.

Two centuries have elapsed since Buffon presented his celebrated needle problem to the French Academy. ${ }^{1}$ A needle of length 1 may assume any position on a grid of parallel lines of distance d apart, where $\mathrm{d}>1$ (Fig. 1). What is the probability $\mathrm{p}$ that the needle will intersect with a line on the grid? The probability of an intersection occurring when the needle lies at any angle $\theta$ to the lines of the grid, $\mathrm{p}(\theta)$

$$
\begin{aligned}
& =\frac{1|\sin \theta|}{\mathrm{d}} \\
\therefore \quad \mathrm{p} & =\frac{2}{\pi} \int_{0}^{\pi / 2} \mathrm{p}(\theta) \mathrm{d} \theta \\
& =\frac{2 l}{\pi \mathrm{d}} \int_{0}^{\pi / 2}|\sin \theta| \mathrm{d} \theta \\
& =\frac{2 l}{\pi \mathrm{d}}
\end{aligned}
$$

*Present address: Department of Pathology, University Medical School, Edinburgh EH8 9AG.

Accepted for publication 17 September 1980

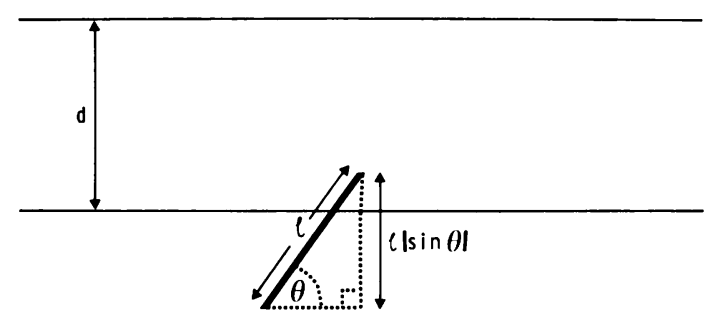

Fig. 1 The Buffon needle problem. A needle of length $l$ may assume any position on a grid of parallel lines of distance $d$ apart, where $d>l$. What is the probability $p$ that the needle will intersect with a line on the grid?

This relationship can be use to estimate the value of $\pi$, but, of more practical significance, it can be generalised to apply to a continuous curve. ${ }^{2}$ An estimate $(\hat{\mathbf{B}})$ of the true length $(\mathrm{B})$ of a curve can be made by counting the number of intersections it makes (I) with the lines of the grid. With the grid shown in Fig. 1,

$$
\widehat{B}=\frac{\pi}{2} \mathrm{I} \mathrm{d}
$$

If the curve assumes $\mathrm{n}$ random positions on the grid and $\widehat{B}$ is calculated using the mean number of intersections for each position (I), namely, 


$$
\widehat{B}=\frac{\pi}{2} \bar{I} d
$$

then $\hat{\mathbf{B}} \rightarrow \mathrm{B}$ as $\mathrm{n} \rightarrow \infty$

With the type of grid used in the present study twice as many intersections are likely to occur so that in this case:

$$
\widehat{\mathrm{B}}=\frac{\pi}{4} \overline{\mathrm{I}} \mathrm{d}
$$

equation 1.

\section{Experiment 1}

In order to demonstrate this relationship, seven pieces of floppy wool measuring from 1 to 7 inches in length were each cast randomly on to the grid 10 times. The mean number of intersections each made with lines on the grid, the standard deviation, and the standard error of the mean (SEM) were calculated for each piece of wool. Figure 2 shows the mean number of intersections $( \pm 2 \times$ SEM) plotted against the length of each piece of wool as originally measured with a ruler. This clearly demonstrates, within the limits of accuracy of this experiment, the direct proportional relationship between the two variables.

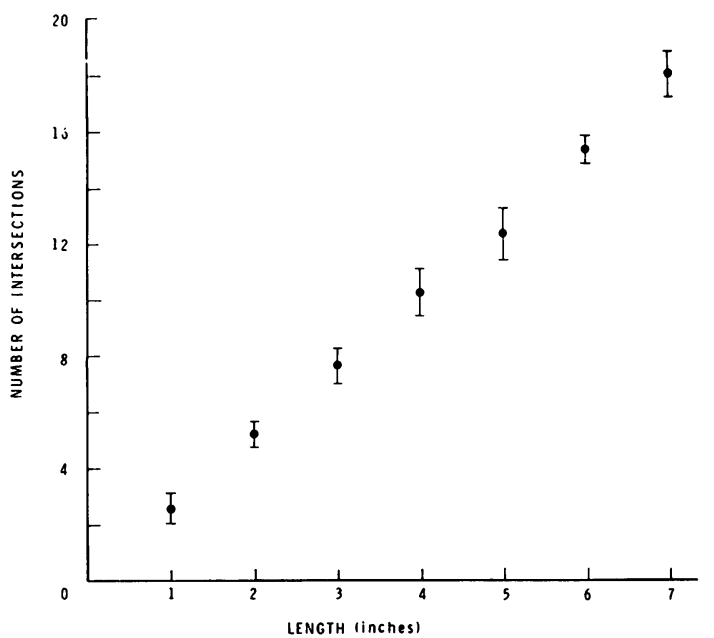

Fig. 2 The mean number of intersections $( \pm 2 \times S E M)$ made by each of seven pieces of floppy wool, 1-7 inches in length, with the lines of the test grid, when each was cast randomly on to the grid 10 times.

The mean number of intersections made by the 7inch piece of wool with the lines of the grid was 18 . By substituting this value of $\bar{I}$ and the value of $d$ ( $\frac{1}{2}$ inch) in equation $1, \widehat{B}$ can be calculated.

$$
\begin{aligned}
\hat{\mathbf{B}} & =\frac{\pi}{4} \overline{\mathbf{I} ~ d} \\
& =\frac{3.14}{4} \times 18 \times \frac{1}{2} \text { inches } \\
& =7.07 \text { inches. }
\end{aligned}
$$

\section{Experiment 2}

There is a property of two-dimensional curves which may be called directional bias. This can be illustrated by regarding a curve (which can be either closed or open) as being made up of a large number of straight line segments of equal length. Each small line segment will be at an angle, $\alpha$, to some given fixed reference line (Fig. 3). Line segments at angles $\alpha$ and $\pi+\alpha$ will have the same direction but opposite sense; since we are not interested in the distinction between two such cases, we shall redefine $\alpha$ (whenever necessary) to lie always in the range $0 \leq \alpha<\pi$.

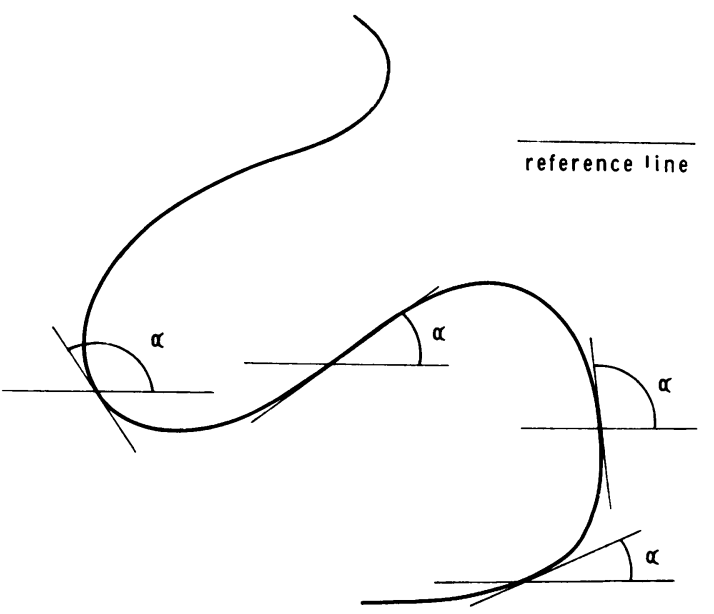

Fig. 3 A curve may be regarded as being made up of a large number of straight line segments of equal length. Each small segment will be at an angle a to some given fixed reference line.

If a frequency distribution curve were constructed for the relative number of straight line segments having directions within each infinitesimal range of angles $\delta \alpha$ between $\alpha=0$ and $\alpha=\pi$ for a particular curve and reference line, this would demonstrate the presence and degree of any directional bias (Fig. 4). Thus a circle has no directional bias, and a straight line has the maximum possible directional bias. An ellipse is biased in the direction of its major axis. More complicated curves, such as the cruciform 
example shown in Fig. 4, may be biased in more than one direction.

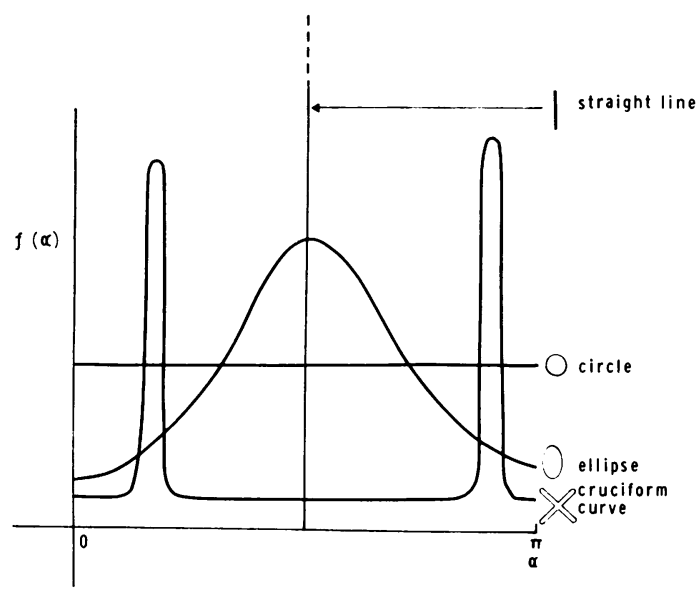

Fig. 4 Four hypothetical frequency distribution curves for the relative number of straight line segments having directions within each infinitesimal range of angles $\delta \alpha$ between $\alpha=0$ and $\alpha=\pi$ for each curve and $a$ reference line, demonstrating the presence and degree of directional bias.

This experiment was designed to explore the effect on length-measurement of the angular orientation of the curve upon the grid. If there is an effect, this will be apparent only with curves that have a directional bias. A circle, for example, in which all directions are equally represented, cannot be affected in this way. On the contrary, a straight line will be maximally affected.

The probable number of intersections $p(I)$ of a straight line of length $B$ with the lines of the grid, at any angle $\theta$ to one of the sets of lines (Fig. 5)

$$
\begin{aligned}
& =\frac{\mathbf{B}|\sin \theta|}{\mathbf{d}}+\frac{\mathrm{B}|\cos \theta|}{\mathbf{d}} \\
& =\frac{\mathbf{B}}{\mathbf{d}}(|\sin \theta|+|\cos \theta|)
\end{aligned}
$$

Figure 6 shows four curves:

Curve 1 is the function:

Curve 2 is

$$
\mathrm{y}=|\sin \theta|
$$

Curve 3 is

$$
y=|\cos \theta|
$$

$$
\mathbf{y}=|\sin \theta|+|\cos \theta|
$$

Curve 4 will be referred to later.

It can be seen from curve 3 that $p(I)$ will vary in an asymmetrical fashion for every $\frac{\pi}{4}\left(45^{\circ}\right)$ change in $\theta$,

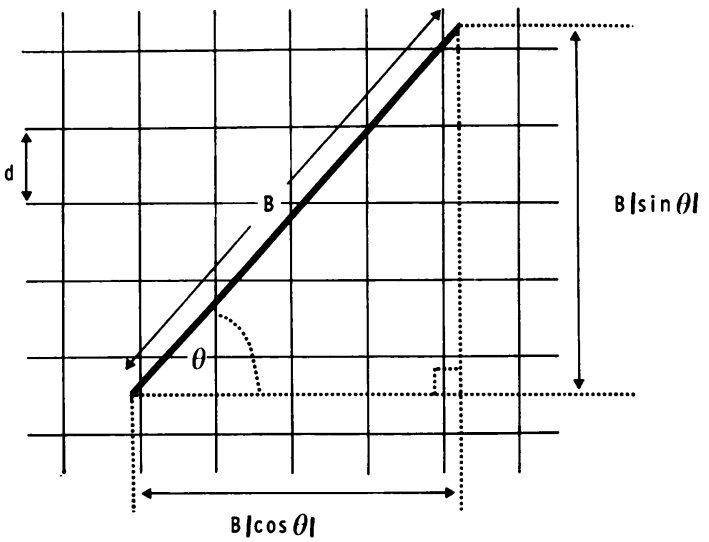

Fig. 5 The probable number of intersections $p(I)$ of a straight line of length $B$ with the lines of the grid, at any angle $\theta$ to one of the sets of lines $=$ $\frac{B|\sin \theta|}{d}+\frac{B|\cos \theta|}{d}$.

beginning at $\theta=0 . \mathrm{p}(\mathrm{I})$ is the product of the con$\operatorname{stant} \frac{\mathrm{B}}{\mathrm{d}}$ and $(|\sin \theta|+|\cos \theta|)$, and $(|\sin \theta|+|\cos \theta|)$ varies asymmetrically from 1 to 1.41 every $45^{\circ}$. This will cause a skew to the right of the frequency distribution of the number of intersections a curve with a directional bias will have with the lines of the grid, as demonstrated in Figure 7. This shows the frequency distribution histogram of the number of intersections made between the grid and an 8-inch straight line cast randomly on to it 505 times.

To demonstrate that this skewness is an effect of angular orientation to the grid, a similar experiment was performed using a curve with much less directional bias. A circle is unsuitable for this purpose because variation in the number of intersections it can make with the grid is very limited and is quite different from that of other curves. A slightly irregular C-shaped curve, with the two limbs of the $\mathrm{C}$ quite close together, was chosen. This curve, which was about 8 inches long, was randomly cast on to the grid 500 times. The number of intersections it made with the grid was counted each time. The resulting frequency distribution histogram is shown in Fig. 8 and this demonstrates no obvious skew.

The effect of the angular orientation of a curve on the grid may be minimised by taking the mean of the number of intersections it makes with the grid at three different angles: $\theta, \theta+30^{\circ}$, and $\theta+60^{\circ}$. Curve 4 in Fig. 6 demonstrates the effect of this in the case of a straight line, being the curve of the function

$$
\begin{gathered}
\mathbf{y}=\frac{1}{3}\left\{[|\sin \theta|+|\cos \theta|]+\left[\left|\sin \left(\theta+30^{\circ}\right)\right|+\right.\right. \\
\left.\left.\left|\cos \left(\theta+30^{\circ}\right)\right|\right]+\left[\left|\sin \left(\theta+60^{\circ}\right)\right|+\left|\cos \left(\theta+60^{\circ}\right)\right|\right]\right\}
\end{gathered}
$$




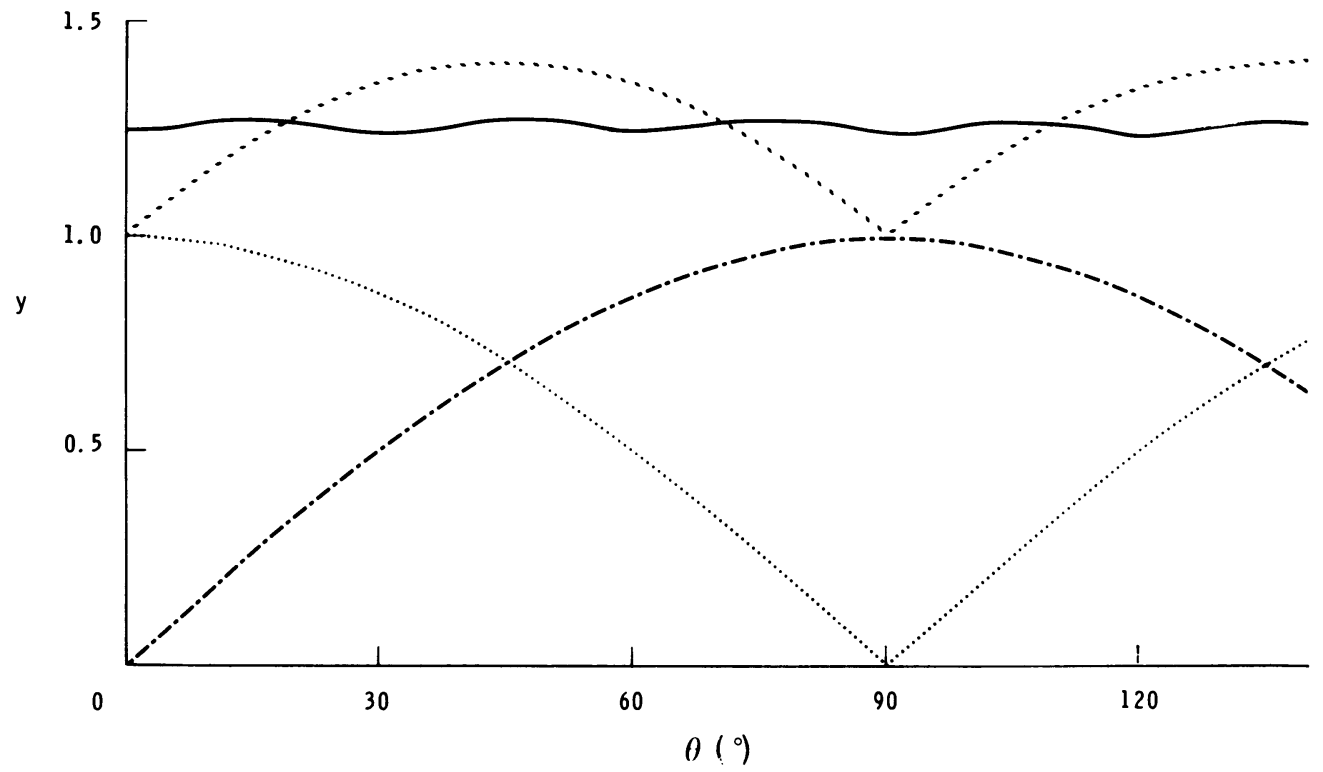

Fig. 6 Four curves representing the functions:

$$
\begin{aligned}
& y=|\sin \theta|-\operatorname{curve} 1(-.-) \\
& y=|\cos \theta|-\operatorname{curve} 2(\ldots .) \\
& y=|\sin \theta|+|\cos \theta|-\operatorname{curve} 3(---) \\
& y=\frac{1}{3}\left\{[|\sin \theta|+|\cos \theta|]+\left[\left|\sin \left(\theta+30^{\circ}\right)\right|+\right.\right. \\
& \left.\left.\left|\cos \left(\theta+30^{\circ}\right)\right|\right]+\left[\left|\sin \left(\theta+60^{\circ}\right)\right|+\left|\cos \left(\theta+60^{\circ}\right)\right|\right]\right\} \\
& -\operatorname{curve} 4(-) .
\end{aligned}
$$

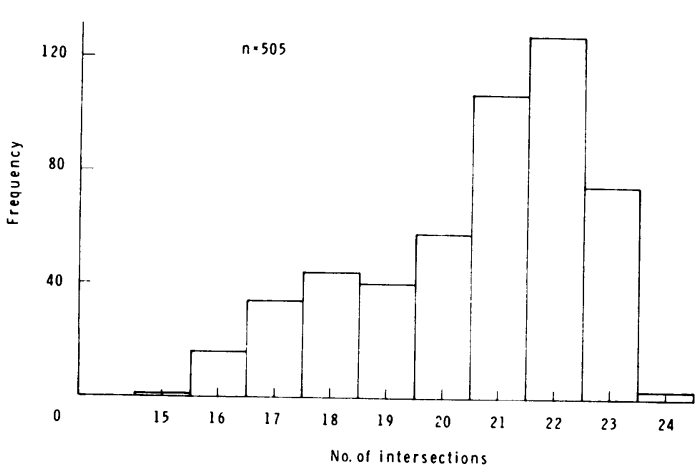

Fig. 7 The frequency distribution histogram of the number of intersections made between the grid and an 8-inch straight line cast randomly on to it 505 times. The predicted skew to the right is demonstrated.

This virtually eliminates the variability due to changes in $\theta$ and therefore the skew imposed by this variability on the frequency distribution.

\section{Experiment 3}

As a demonstration of the ability of the grid to measure the length of lines of various shapes, seven

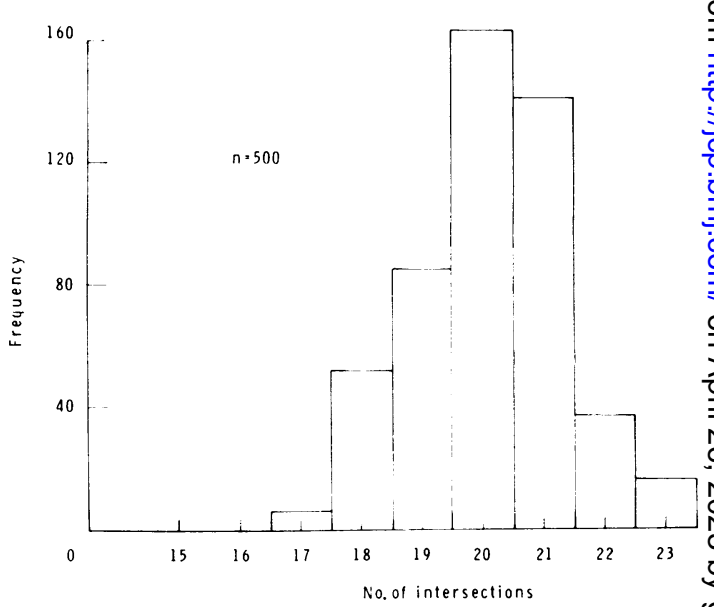

Fig. 8 The frequency distribution histogram of the number of intersections made between the grid and $a$ curve with little directional bias cast randomly on to it 500 times. Compared to Fig. 7, the variability is less and the skew to the right has disappeared.

lines, of the same length but of different shapes, were traced from the same length of wool on too 
clear plastic. Each line was then randomly cast on to another sheet of clear plastic pivoted centrally over the grid and marked off in $5^{\circ}$ intervals around the pivot point. The number of intersections each line made with the lines of the grid was counted at the original position and again after rotating the pivoted sheet of plastic $30^{\circ}$ and $60^{\circ}$ from the original position. This process was repeated 10 times for each line, resulting in a total of $\mathbf{3 0}$ measurements for each line, the mean of which would be minimally affected by angular orientation to the grid. The mean and standard deviation were determined and are shown in Figure 9. The mean and standard deviation of each set of observations were closely similar in spite of the great variability in the shape of the lines.

\begin{tabular}{|c|c|c|c|}
\hline Curve & Shape & $\begin{array}{l}\text { Mean number } \\
\text { of intersections }\end{array}$ & $\begin{array}{l}\text { Standard } \\
\text { deviation }\end{array}$ \\
\hline 1 & & 20.5 & $1 \cdot 2$ \\
\hline 2 & & 20.7 & 1.5 \\
\hline 3. & & 20.3 & 1.5 \\
\hline 4 & & $20 \cdot 1$ & 1.5 \\
\hline 5 & & 20.8 & $1 \cdot 2$ \\
\hline 6 & & $21 \cdot 2$ & 1.7 \\
\hline 7 & & 20.0 & 15 \\
\hline
\end{tabular}

Fig. 9 Length of various shaped curves of similar length in terms of the number of intersections with the lines of the grid (30 sets of observations for each curve).

Using the data from this experiment, it is possible to estimate the number of random observations that it is necessary to make in order to achieve a particular degree of accuracy of length measurement. The data suggest that a standard deviation (s) of about 1.5 would be expected when the mean number of intersections $(\overline{\mathbf{x}})$ is 20 . The $95 \%$ confidence limits for the true mean $\mu$, assuming normal distribution of the data, are:

$$
\overline{\mathrm{x}}-\frac{1.96 \sigma}{\sqrt{\mathrm{n}}}<\mu<\overline{\mathrm{x}}+\frac{1.96 \sigma}{\sqrt{\mathrm{n}}}
$$

where $\sigma$ is the standard deviation of the population and $\mathrm{n}$ is the number of observations.

In order to be $95 \%$ confident that $\mu$ lies within $\pm 5 \%$ of $\bar{x}$

$$
\begin{aligned}
& \qquad \frac{1.96 \sigma}{\sqrt{n}} \text { must equal } 5 \% \text { of } \bar{x} \\
& \text { therefore } \frac{1.96 \sigma}{\sqrt{n}}=\frac{\bar{x}}{20}
\end{aligned}
$$

The true value of $\sigma$ is not known, so s is used as the nearest approximation, as follows:

$$
\frac{1.96 \mathrm{~s}}{\sqrt{\mathrm{n}}}=\frac{\overline{\mathrm{x}}}{20}
$$

Substituting the data derived from experiment 3

$$
\frac{1.96 \times 1.5}{\sqrt{\mathrm{n}}}=\frac{20}{20}
$$

therefore $\sqrt{\mathrm{n}}=1.96 \times 1.5$

therefore $\mathrm{n}=8.6$

Thus nine sets of observations should yield a mean number of intersections which is within $5 \%$ of the true population mean.

\section{Conclusions}

The grid used in the present study is clearly an effective device for measuring the length of lines of various shapes.

The measurement of the length of curves with directional bias is influenced by the angular orientation of the curve on the grid. Directional bias causes a skew to the right in the frequency distribution and increases the variability. This effect can be virtually eliminated by taking the mean of three measurements, of which the second and third are taken with the curve rotated $30^{\circ}$ and $60^{\circ}$ respectively from its original position on the grid. If the effects of angle are minimised in this way, an estimate of the length of a curve made from the mean of nine or more measurements should be within $5 \%$ of its true length (95\% confidence limits), if this is such that the mean number of intersections is about 20 .

This information has been used to modify a method of estimating the density of gastrin cells in tissue sections and to identify and quantify one source of variability inherent in the method.

We thank Smith, Kline, and French Laboratories Ltd, Welwyn Garden City, for generously supporting one of us (RM) during the present study, and Dr SC Truelove for his invaluable help in the preparation of this paper.

\section{References}

${ }^{1}$ Buffon G. Essai d'arithmétique morale. Supplément à l'Histoire Naturelle 1777;4.

2 Kendall MG, Moran PAP. Geometrical probability. London: Charles Griffin, 1963.

Requests for reprints to: Mr RLE McIntyre, Monash University Department of Surgery, Prince Henry's Hospital, Melbourne, Australia, 3004. 